\title{
Effects of concentration of Jerusalem artichoke powder on the quality of artichoke-enriched bread fermented with mixed cultures of Saccharomyces cerevisiae, Torulaspora delbrueckii JK08 and Pichia anomala JK04
}

\author{
Agung Wahyono1, Sae-Byuk Lee', Soo-Hwan Yeo², Woo-Won Kang ${ }^{3}$, Heui-Dong Park ${ }^{\text {* }}$ \\ ${ }^{1}$ School of Food Science and Biotechnology, Kyungpook National University, Daegu, Korea, ${ }^{2}$ Department of Agro-food Resource, National \\ Academy of Agricultural Science, RDA, Chonju, Korea, ${ }^{3}$ Department of Food \& Food-service Industry, Kyungpook National University, \\ Sangju, Korea
}

\section{A B S T R A C T}

\begin{abstract}
A co-culture of Torulaspora delbrueckii JK08 and Pichia anomala JK04 in combination with commercial baker's yeast (Saccharomyces cerevisiae) was used as a leavening agent in the preparation of Jerusalem artichoke powder (JAP)-enriched bread. The breads were made by substituting of $5,7.5$, and $10 \%$ wheat flour with JAP. Water absorption decreased with increasing JAP. JAP enrichment of $5 \%$ resulted adverse effect on dough development time, mixing tolerance index, and dough stability, but comparable to those in control bread at a higher dose. The use of a co-culture and JAP enrichment simultaneously decreased specific volume, but improved bread yield. The co-culture was able to slow the darkening effect of bread crumb imparted by JAP enrichment at a dose of 5 and $7.5 \%$. Hardness and chewiness were worsened by the use of a co-culture and JAP enrichment, while springiness and cohesiveness were improved. The cell density and mean cell size of bread crumb were improved by the use of a co-culture, while cell area fraction was unaltered except at $10 \%$ of JAP-enrichment. In general, the use of a co-culture improved the consumer preference of JAP-enriched bread at a dose of 5 and $7.5 \%$. Thus, we suggest the use of co-culture in bread making enriched with JAP for improved bread quality.
\end{abstract}

Keywords: Bread making; Jerusalem artichoke; Mixed culture; Pichia anomala; Torulaspora delbrueckii

\section{INTRODUCTION}

Consumer awareness of healthy bread products is greatly increasing because of the high prevalence of diseases of civilization, such as obesity, diabetes, and coronary heart disease. This has led to an increasing interest in the manufacture of breads for special dietary concerns and increased nutritional value (Hager et al., 2012). It is well known that dietary fiber consumption may reduce coronary heart-related diseases and diabetes incidence (Wang, 2002), as well as increase mineral absorption, improve the immune response, and play a role in colorectal cancer prevention (Morris and Morris, 2012). As determined by the Institute of Medicine, National Academy of Sciences, USA, the Adequate Intake (AI) of total dietary fiber for children, adolescents, and adults was set to $14 \mathrm{~g}$ dietary fiber/1000 $\mathrm{kcal}$, to reduce the chronic diseases (Cho and Samuel,
2009). However, total dietary fiber intake remains below the recommended level (Roberfroid, 2002). The specific effects of fiber intake are not always clearly visible or easy to explain because the fiber complex is multifunctional (Salovaara et al., 2007). In addition, previous studies have shown that fiber enrichment can have detrimental effects on bread quality, influencing consumer preference (Morris and Morris, 2012; Skendi et al., 2010; Rubel et al., 2015). Through experimentation, new applications, and product and process development, scientists and industries have attempted to help consumers increase fiber intake in a convenient manner (Salovaara et al., 2007).

The use of Jerusalem artichoke (JA) (Helianthus tuberosus) for food, fuel production, and industrial products has increased significantly. This crop has high tuber yield, grows well in poor soils, and is resistant to cold temperature, pests, and

\footnotetext{
*Corresponding author:

Heui-Dong Park, School of Food Science and Biotechnology, Kyungpook National University, 80 Daehak-ro, Buk-gu, Daegu, 702-701, Korea. Tel.: +82-53-950-5774; Fax: +82-53-950-6772. E-mail: hpark@knu.ac.kr
} 
diseases (Kosaric et al., 1984). The tuber of JA is a good source of inulin, with its content ranging from $7-30 \%$ of fresh weight (Kays and Nottingham, 2008). In dry tubers, the inulin content ranges from $75-85 \%$ and its degree of polymerization is 6-14 (Gunnarsson et al., 2014).

Various studies on baking products have been conducted to examine the use of JA and its derivatives. However, the quality of bread enriched with these materials is far from optimal. Fiber enrichment can have detrimental effects on bread quality such us smaller loaves, a harder crumb and darker color (Morris and Morris, 2012; Skendi et al., 2010), as well as a decreased specific volume and cell (pore) area ratio (Rubel et al., 2015). However, improving the quality of enriched-bread is feasible through process innovations. It has been reported that the addition of plasma bovine protein and inulin produced smaller and homogenous air cells and comparable hardness relative to control bread (Furlán et al., 2015). Moreover, the enrichment of wheat bread with 15\% fermented JA and lactic acid bacteria does not impair bread quality or safety (Bartkiene et al., 2013). There is a need to conduct additional studies to examine bread enriched with JA and its derivatives without compromising consumer acceptance.

The role of yeast in bread making is paramount and greatly affects bread quality. The yeast gassing rate is critical for leavening the dough and contributes to the flavor and crumb structure of bread. The gassing rate is influenced by the dough formulation, specific fermentation parameters, and particularly the yeast intrinsic traits (Randez-Gil et al., 1999). Recently, the use of co-cultured yeast as a leavening agent has been reported to confer favorable effects in bread making, such as improved bread quality and delayed staling (Elhariry et al., 2011), enhanced nutritional content and shelf life of cassava-wheat bread (Ogunbanwo et al., 2008), and enhanced aroma (Annan, 2003).

There have been no reported studies on the use of cocultured yeast to produce fiber-enriched bread. Previous studies have employed conventional yeast, Saccharomyces cerevisiae, as the leavening agent (Rubel et al., 2015; Bartkiene et al., 2013; Peressini and Sensidoni, 2009; Furlán et al., 2015). We found that co-culture with S. cerevisiae, T. delbrueckii JK08, and/or P. anomala JK04 was superior to single-culture with $S$. cerevisiae in nearly all quality parameters in wheat bread (Wahyono et al., In press). Here, we used a co-culture of T. delbrueckii JK08 and $P$. anomala JK04 in combination with commercial dry yeast as a leavening agent and investigated its effects on the quality of Jerusalem arthicoke-enriched bread. The physical properties, structural crumb features, textural profile, sensorial properties, and the correlation among these parameters were examined.

\section{MATERIALS AND METHODS}

\section{Materials}

Torulaspora delbrueckii JK08 (TD) and P. anomala JK04 (PA) were obtained from the Institute of Fermentation Biotechnology, Kyungpook National University, Daegu, South Korea. The strains were sub-cultured in yeast extractpeptone-dextrose (Difco, Franklin Lakes, NJ, USA) agar (Oxoid, Hampshire, UK) and stored at $4{ }^{\circ} \mathrm{C}$. Commercial baker's yeast containing $S$. cerevisiae (SC) was compressed instant baker's yeast (saf-instant S.I., Lesaffre, Marcq, France). Commercial wheat flour (Beksul, CheilJedang, Seoul, South Korea) contained $15.39 \%$ moisture, 13.93\% protein, $0.47 \%$ ash, with a $\mathrm{pH}$ of 4.35 . JA powder (JAP) contained $49.50 \%$ inulin, $3.25 \%$ moisture, $4.88 \%$ ash, $3.30 \%$ free sugar and $0.44 \%$ crude lipid was purchased online (http://jangmung.com). Sugar and salt were purchased from a supermarket in Sangju, South Korea.

\section{Propagation of yeast strains}

The strains were sub-cultured in 5\% yeast extract-peptonedextrose broth and cultivated in a rotary shaker (JSSI$300 \mathrm{C}$, JS Research, Gongju, South Korea) at $30{ }^{\circ} \mathrm{C}$ for $48 \mathrm{~h}$ with shaking at $180 \mathrm{rpm}$. Yeast cells were collected by centrifugation (Hanil Supra 22K, Hanil, Incheon, South Korea) at $4000 \times g$ for $10 \mathrm{~min}$ at $4{ }^{\circ} \mathrm{C}$. The supernatant was discarded and the pellet was re-suspended in distilled water, vortexed thoroughly, and stored at $4{ }^{\circ} \mathrm{C}$. Cell density was measured using a hemocytometer (Neubauer chamber, Celeromics, Cambridge, UK) as described previously (Wahyono et al., 2015).

\section{Flour properties}

The properties of blended flour were determined using a farinograph (Brabender, Duisburg, Germany) (AACC 54-2, 2000). The blended flour was mixed well. Approximately $300 \mathrm{~g}$ was placed into the corresponding farinograph mixing bowl. Water was added from a burette to the flour and mixed to form a dough. As the dough formed, the resistance of the mixing blades increased, and the pen recorded dough movement continuously on a chart to form a farinogram. To ensure that farinograms from different samples could be compared, the midpoint of the farinograph bandwidth at the maximum resistance was always centered on the 500 brabender unit (BU) line by adjusting the water used. Next, water absorption, dough development time, mixing tolerance index, and stability were recorded.

\section{Bread making procedure}

Bread making was performed by enrichment with JAP and the mixed culture as a leavening agent. JAP enrichment was $0,5,7.5$, and $10 \%$, based on the weight of wheat flour. The leavening agent included co-cultured $T$. delbrueckii 
JK08 and P. anomala JK04 in combination with commercial baker's yeast. For the mixed culture, the co-culture was adjusted to replace approximately $50 \%$ of the commercial baker's yeast. Bread making was conducted as follows: 1) wheat flour without JAP enrichment and leavened using commercial baker's yeast (SC-0), 2) wheat flour without JAP enrichment and leavened using mixed culture (MC-0), 3) wheat flour with 5\% JAP enrichment and leavened using mixed culture (MC-5), 4) wheat flour with 7.5\% JAP enrichment and leavened using mixed culture (MC-7.5), and 5) wheat flour with 10\% JAP enrichment and leavened using mixed culture (MC-10). Water content was adjusted considering the farinograph water absorption value. Bread formulations are shown in Table 1. Baking was carried out in a bread maker (National SD-BT102, Panasonic, Osaka, Japan) using 4-h bread making setting. Baking was performed in triplicate. After baking, the bread loaves were tempered at ambient temperature $\left(28-30{ }^{\circ} \mathrm{C}\right)$ before analysis.

\section{Physical properties of bread}

The moisture content of the bread crumb was determined using the oven-drying method (Czuchajowska et al., 1989). Specific volume was determined using the seed displacement method (AACC 10-05, 2000). Bread yield efficiency was calculated as described by Movahed et al. (2012). The crust and crumb colors were measured using the CR-400 Chroma Meter (Konica-Minolta, Tokyo, Japan); Hunter's $L$ (lightness), $a$ (redness), and $b$ (yellowness) values were measured for six regions of bread crust and crumb (Wahyono et al., 2015). The whiteness index (WI) was calculated according to Hsu (2003) and Lin et al. (2009).

\section{Texture profile analysis}

Texture profile analysis was carried out in triplicate for three slices of bread using a texture analyzer (CT3 4500, Brookfield, Middleboro, MA, USA). Bread samples were sliced to approximately $25-\mathrm{mm}$ thickness. Hardness, chewiness, cohesiveness, and springiness of the center of the bread slices were measured. The settings and conditions were as described previously (Wahyono et al., In press):

Table 1: Bread formulations

\begin{tabular}{lccccc}
\hline Ingredients & \multicolumn{5}{c}{ Sample } \\
\cline { 2 - 6 } & SC-0 & MC-0 & MC-5 & MC-7.5 & MC-10 \\
& $(\mathbf{0} \%)$ & $(5 \%)$ & $(7.5 \%)$ & $(\mathbf{1 0} \%)$ \\
\hline Wheat flour (g) & 280 & 280 & 266 & 259 & 252 \\
Jerusalem artichoke powder (g) & - & - & 14 & 21 & 28 \\
Sugar (g) & 16.8 & 16.8 & 16.8 & 16.8 & 16.8 \\
Salt (g) & 5.6 & 5.6 & 5.6 & 5.6 & 5.6 \\
Water (mL) & 200 & 200 & 195 & 190 & 185 \\
Yeast; & & & & & \\
S. cerevisiae (g) & 2.8 & 1.4 & 1.4 & 1.4 & 1.4 \\
T. delbrueckii JK08 (cells) & - & $5 \times 10^{10}$ & $5 \times 10^{10}$ & $5 \times 10^{10}$ & $5 \times 10^{10}$ \\
P. anomala JK04 (cells) & - & $5 \times 10^{10}$ & $5 \times 10^{10}$ & $5 \times 10^{10}$ & $5 \times 10^{10}$ \\
\hline
\end{tabular}

the acrylic cylindrical probe had a 38.1-mm diameter, the pretest speed was $2 \mathrm{~mm} / \mathrm{s}$, the test speed was $2 \mathrm{~mm} / \mathrm{s}$, the post-test speed was $2 \mathrm{~mm} / \mathrm{s}$, the distance was $10 \mathrm{~mm}$, and the trigger load was $50 \mathrm{~g}$.

\section{Bread crumb image analyses}

The structural features of the bread crumb were analyzed using the Image J software $(1.47 \mathrm{v}$, National Institutes of Health, Bethesda, MD, USA). Structural features included bread cell density, mean cell area, cell area relative distribution, and the fraction of cell area to total area. Bread crumb images were captured using a scanner (Epson Perfection V370 Photo, Epson, Suwa, Japan) at a resolution of $800 \times 800$ dpi. Images were calibrated to reflect the actual size using a known scale and cropped to $60 \times 60 \mathrm{~mm}$, filtered using a bandpass filter, and converted into binary images using the convert to mask feature for differentiating between the cell and non-cell area. The threshold was adjusted using a black and white and a dark background. Before analyzing particles (cells), the particle size was set from $0.01 \mathrm{~mm}^{2}$ to infinity and the circularity was set from 0 to 1 (Wahyono et al., In Press). The results were then tabulated and calculated using Microsoft Excel 2007v.

\section{Sensory evaluation}

Bread samples were prepared from a freshly baked loaf (6-8 $\mathrm{h}$ after baking). The bread samples were cut into approximately $50 \times 20 \times 25 \mathrm{~mm}$ sections and served on a small paper plate. Sensory evaluations were conducted by 15 semi-trained consumers who were students at Kyungpook National University, South Korea. The sensory attributes tested included texture, color, flavor, mouthfeel, and overall acceptability. The sensory attributes scale used to assess the bread was as follows: 1 , extremely dislike; 4 , neither like nor dislike; and 7, extremely like (Ulziijargal et al., 2013).

\section{Statistical analysis}

To examine statistical significance, the data were analyzed using one-way analysis of variance followed by Duncan's multiple range test at $p<0.05$ level of significance. The correlation between parameters was analyzed using 2-tailed Pearson correlation at $p<0.05$. Analysis was carried out using SPSS for windows (ver. 19, SPSS, Inc., Chicago, IL, USA). The graphs were constructed using Microsoft Excel 2007v.

\section{RESULTS AND DISCUSSION}

\section{Flour properties}

JAP enrichment altered the flour properties measured by farinograph as shown in farinogram (Fig. 1). Increased JAP decreased water absorption. Wang (2002) and O'Brien et al. (2003) also reported decreased water absorption with 
the addition of inulin powder. Lower water binding ability of inulin resulted in lower hydration of the structureforming hydrocolloids (Juszczak et al., 2012), which may have reduced water absorption. Dough development time (DDT) and mixing tolerance index (MTI) were altered with increasing JAP. At 5\% JA enrichment, DDT was shortened (8 min), while MTI was markedly increased (80 BU). At a higher dose, DDT was increased and MTI was decreased. Dough stability decreased when 5\% JAP was used (12.5 min), which subsequently improved in the presence of $7.5 \%$ JAP (14 min) and then recovered at 10\% JAP (24 min).

DDT is the time between the origin of the curve and its maximum. MTI is measured as the difference in BU between the top of the optimum point on the curve and the point $5 \mathrm{~min}$ later. Stability is defined as the difference in minutes between the arrival time and the time the top of the curve falls below the $500 \mathrm{BU}$ line. The flour with higher absorption, long development time, and more tolerance to over-mixing is generally considered to be a good ingredient for bread making (Atwell, 2001). Thus, JAP enrichment can be beneficial or detrimental to bread making depending on the amount of JAP used.

\section{Physical properties of bread}

The use of co-culture and JAP enrichment significantly influenced the physical properties of bread as shown in Table 2. The co-culture did not modify the crumb moisture of non-enriched bread (MC-0) relative to the control bread (SC-0), but increased JAP significantly reduced the crumb moisture. This was in accordance with a decreased in water absorption when JAP was increased (Fig. 1). Wang et al. (2002) reported that inulin addition reduced crumb moisture. In contrast, Rubel et al. (2015) reported a comparable moisture content between control and JA inulin-enriched bread. During baking, the crust forms a resistant barrier, retaining moisture, which had been absorbed previously into the dough (Stear, 1990). Therefore, the final moisture content of bread was largely determined by water absorption during dough preparation and by water loss during baking (Rubel et al., 2015).

The specific volume of JAP-enriched bread was significantly affected by the use of co-cultured $T$. delbrueckii JK08 and P. anomala JK04. The co-culture significantly reduced bread specific volume relative to the control bread (Tabel 2). We previously found that T. delbrueckii JK08 and P. anomala JK04 had lower leavening abilities and slower growth than $S$. cerevisiae, resulting in a lower specific volume (Wahyono et al., 2015). JAP enrichment modified the bread specific volume depending on the dose used. At a dose of $5 \%$, JAP enrichment improved bread specific volume. This was because lower water content in JAP bread produced

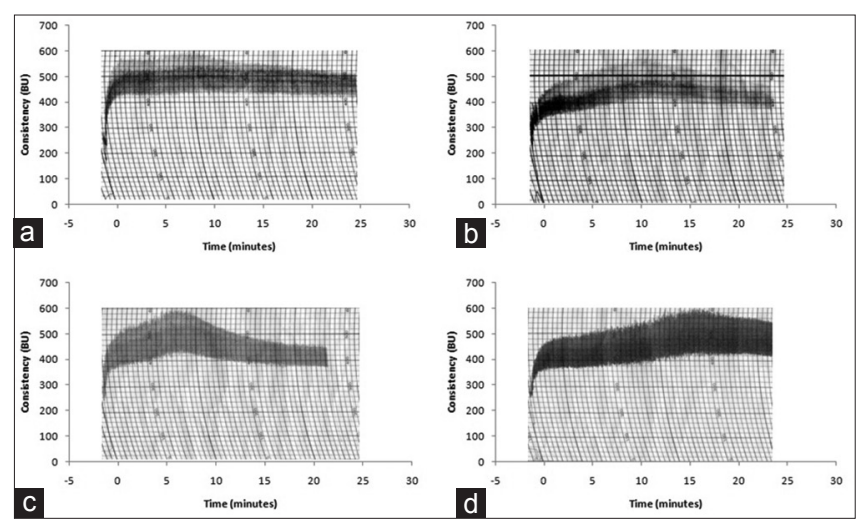

Fig 1. Farinogram of wheat flour enriched with different dose of Jerusalem artichoke powder (JAP). (a) without JAP; (b) JAP 5\%; (c) JAP 7.5\%; (d) JAP 10\%. BU brabender unit

Table 2: Physical properties of bread made with JAP and leavened using co-culture

\begin{tabular}{lccc}
\hline Samples & $\begin{array}{c}\text { Crumb moisture } \\
\text { content }(\%)\end{array}$ & $\begin{array}{c}\text { Specific volume } \\
\left(\mathbf{c m}^{3} / \mathbf{g}\right)\end{array}$ & $\begin{array}{c}\text { Bread yield } \\
\text { efficiency }(\%)\end{array}$ \\
\hline SC-0 (control) & $47.39 \pm 0.15 \mathrm{a}$ & $4.45 \pm 0.09 \mathrm{~b}$ & $143.87 \pm 0.48 \mathrm{~b}$ \\
MC-0 & $47.12 \pm 0.14 \mathrm{a}$ & $4.05 \pm 0.09 \mathrm{c}$ & $150.35 \pm 1.33 \mathrm{a}$ \\
MC-5 & $45.59 \pm 0.93 \mathrm{bc}$ & $4.63 \pm 0.10 \mathrm{a}$ & $142.47 \pm 1.22 \mathrm{~b}$ \\
MC-7.5 & $45.95 \pm 0.18 \mathrm{~b}$ & $3.94 \pm 0.12 \mathrm{c}$ & $149.90 \pm 0.18 \mathrm{a}$ \\
MC-10 & $44.96 \pm 0.22 \mathrm{c}$ & $3.54 \pm 0.06 \mathrm{~d}$ & $149.31 \pm 0.26 \mathrm{a}$ \\
\hline
\end{tabular}

Values are the mean of the three replicates \pm SD

greater specific volume. Furthermore, the bread specific volume decreased as JAP dose increased (7.5\% and 10\%). A lower JA inulin addition had no significant effect on the storage or elastic modulus $\left(G^{\prime}\right)$ of the dough, but a greater dose decreased elastic modulus. This was attributed to disruption of the starch-gluten matrix, which impaired gas retention and decreased bread specific volume and cell/total area ratio (Rubel et al., 2015). Peressini and Sensidoni (2009) reported that increased inulin increased solid-like behavior, preventing the expansion of dough during the fermentation stage and significantly reducing bread volume.

The use of a co-culture significantly increased the yield of non-enriched bread (MC-0), while JAP enrichment increased the yield of bread only at a greater dose (MC-7.5 and MC-10). Our previous study demonstrated that the use of a single culture of T. delbrueckii JK08 or P. anomala JK04 as a leavening agent significantly increased bread yield compared to that using S. cerevisiae (Wahyono et al., 2015). Similarly, an inulin addition of $8 \%$ increased bread yield. This may be attributed to the binding of water to inulin, which was indicated by the values of total baking loss and was inversely related to bread yield (Korus et al., 2006).

The use of co-culture and JAP enrichment significantly affected the color properties of bread as shown in Table 3 . The difference in color among treated bread was detected 
Wahyono, et al.: Jerusalem artichoke powder enriched-bread

Table 3: Chromaticity of bread crust and crumb made with JAP and leavened using co-culture

\begin{tabular}{|c|c|c|c|c|c|c|c|c|}
\hline \multirow[t]{2}{*}{ Samples } & \multicolumn{4}{|c|}{ Crust color } & \multicolumn{4}{|c|}{ Crumb color } \\
\hline & $\mathbf{L}$ & a & b & $\begin{array}{l}\text { Whiteness } \\
\text { index (WI) }\end{array}$ & $\mathbf{L}$ & a & b & $\begin{array}{l}\text { Whiteness } \\
\text { index (WI) }\end{array}$ \\
\hline SC-0 (control) & $42.01 \pm 1.55 a$ & $6.41 \pm 0.16 c$ & $18.62 \pm 0.78 a$ & $38.75 \pm 1.22 \mathrm{a}$ & $50.95 \pm 2.02 b$ & $-2.20 \pm 0.10 c$ & $7.06 \pm 0.24 \mathrm{e}$ & $50.39 \pm 1.95 b$ \\
\hline $\mathrm{MC}-0$ & $41.29 \pm 0.74 a$ & $7.97 \pm 0.23 b$ & $19.02 \pm 0.36 a$ & $37.77 \pm 0.62 a$ & $56.84 \pm 0.53 a$ & $-2.73 \pm 0.01 d$ & $9.20 \pm 0.17 d$ & $55.79 \pm 0.51 a$ \\
\hline MC-5 & $33.22 \pm 1.36 b$ & $8.51 \pm 1.08 a b$ & $14.75 \pm 1.12 b$ & $31.06 \pm 0.94 b$ & $49.05 \pm 1.53 b$ & $-1.70 \pm 0.08 b$ & $10.01 \pm 0.07 c$ & $48.05 \pm 1.49 b$ \\
\hline MC-7.5 & $32.79 \pm 1.84 b$ & $8.82 \pm 0.27 a b$ & $14.48 \pm 0.99 b$ & $30.67 \pm 1.55 b$ & $49.71 \pm 0.71 b$ & $-1.69 \pm 0.05 b$ & $11.26 \pm 0.30 b$ & $48.43 \pm 0.69 b$ \\
\hline MC-10 & $30.75 \pm 2.43 b$ & $9.33 \pm 0.44 a$ & $13.44 \pm 1.56 b$ & $28.83 \pm 2.00 b$ & $45.90 \pm 1.24 c$ & $-1.05 \pm 0.12 a$ & $12.34 \pm 0.08 a$ & $44.50 \pm 1.19 c$ \\
\hline
\end{tabular}

Values are the mean of the three replicates \pm SD
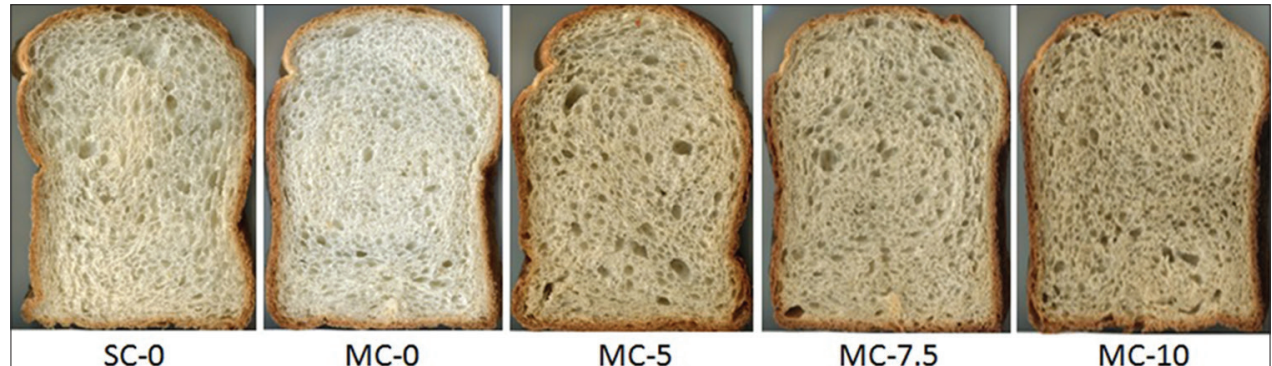

Fig 2. Digital images of bread crumb enriched with JAP and leavened using co-culture

in bread crumb by simple observation as shown in Fig. 2 . Without JAP, the co-culture did not significantly affect the crust lightness or yellowness and whiteness index, but affected redness relative to the control bread. JAP enrichment significantly decreased crust lightness and yellowness and whiteness index, but increased redness. Further, increased JAP did not significantly alter these parameters. Rubel et al. (2015) demonstrated that at a lower dose $(2.5 \%)$, JA inulin addition enhanced the lightness of bread crust but decreased redness and yellowness. At a greater dose (5\%), JA inulin significantly decreased crust lightness, and the decrease in redness or yellowness was more pronounced. Similarly, Hager et al. (2011) reported that the inulin addition of 9\% resulted in a darker crust on enriched gluten-free or wheat bread. The darker crust color of inulin-enriched bread has been attributed to the Maillard reaction. As the dose of inulin is increased, the rate of the Maillard reaction is increased. During baking, the fructan chains of inulin were degraded to produce simpler sugars, which may have contributed to the Maillard reaction and caramelization of the crust (Poinot et al., 2010).

The use of the co-culture significantly improved the crumb lightness and whiteness index of non-enriched bread (MC-0) relative to the control (SC-0). Meanwhile, JAP enrichment impaired the crumb color by decreasing lightness and whiteness index, and increased crumb redness and yellowness. However, the co-culture was able to decrease a darkening effect given by JAP (5 and 7.5\%) on the bread crumb, resulting in a color comparable to that of the control bread. At the highest dose (10\%), a darkening effect was more pronounced and crumb color was significantly impaired. Rubel et al. (2015) reported a significant darkening effect on bread crumb with the addition of $5 \% \mathrm{JA}$ inulin. Our previous study demonstrated that using $P$. anomala JK04 alone as a leavening agent significantly brightened the bread crumb relative to that using $S$. cerevisiae (Wahyono et al., 2015). The brightening effect on bread crumb was also consistent when $P$. anomala JK04 and $S$. cerevisiae were used as a co-culture (Wahyono et al., In press).

\section{Texture profiles of bread}

The use of the co-culture insignificantly increased hardness or chewiness of non-enriched bread (MC-0), but significantly improved springiness or cohesiveness relative to the control bread (SC-0). Increased JAP significantly increased hardness and chewiness. It seems that the use of co-culture and JAP enrichment simultaneously enhanced crumb hardness and chewiness. The springiness or cohesiveness improved with JAP enrichment; all doses of JAP showed similar effects (Fig. 3).

Rubel et al. (2015) reported an increase in hardness, chewiness, and cohesiveness in bread crumb with increased addition of JA inulin. Springiness was unchanged with the addition of 5\% JA inulin. Similarly, Korus et al. (2006) reported increased hardness in gluten-free bread relative to control bread with the addition of $8 \%$ inulin. In contrast, the addition of inulin reduced crumb cohesiveness and springiness. In addition, Hager et al. (2011) observed an increased crumb hardness as well as an increased rate of staling with the incorporation of inulin. The hardening effect on bread crumb could be attributed to the fact that a significant amount of water is bounded by the 


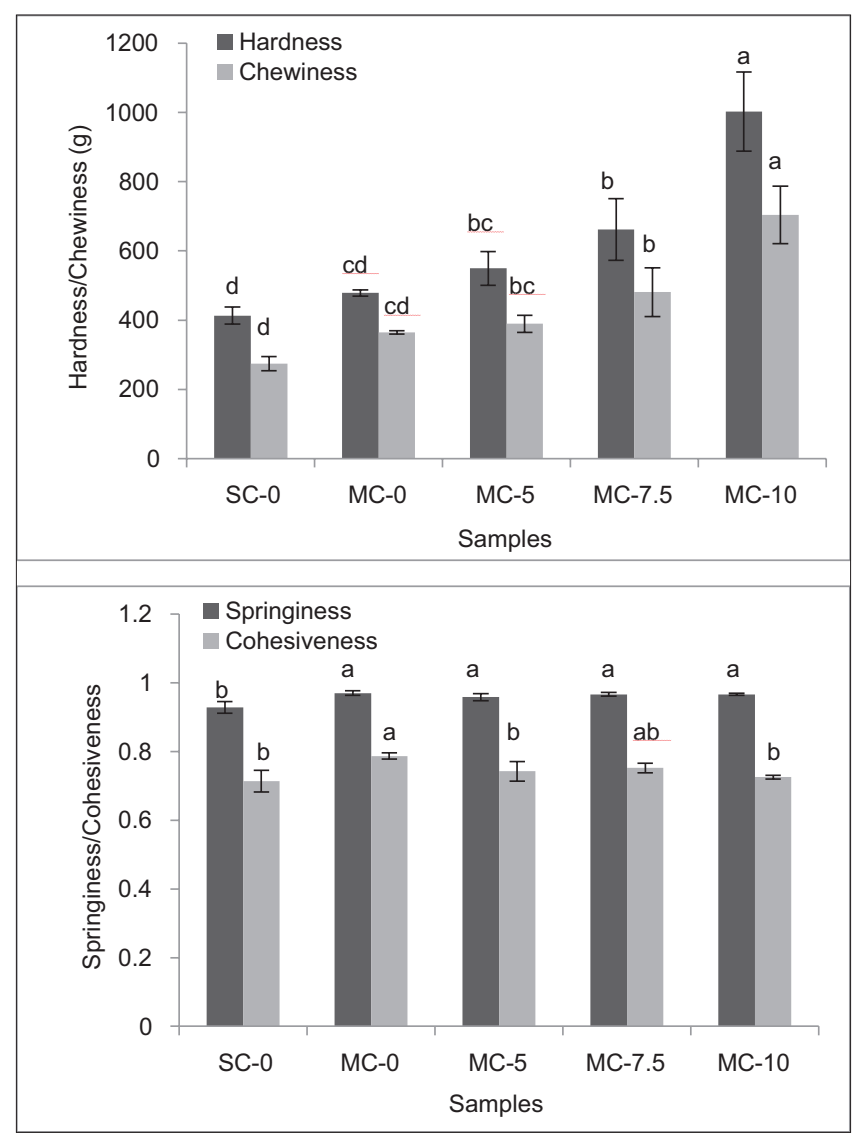

Fig 3. Texture profiles of JAP-enriched bread leavened with coculture. Different letters among textural parameters reflect significant differences at the level $p<0.05$

polysaccharide, in turn affecting the development of the gluten network. This strongly affects the dough properties. It has been reported that at a greater dose, inulin addition decreased elastic modulus, causing a decrease in bread specific volume and cell/total area ratio (Rubel et al., 2015), and consequently increased crumb hardness.

Hardness and chewiness are considered to be negative attributes of most baked products. A hardy or a chewy bread generally requires considerable chewing force or time. In contrast, springiness and cohesiveness are positive attributes. Cohesiveness is concurrently controlled by moisture content and the strength of the network surrounding the cells in the crumb. Greater cohesiveness reflects a better texture of bread crumb, as it is expected to readily form a ball in the mouth. Springiness is related to the strength of the crumb cell wall network. Thus, a fresh bread crumb is expected to have greater springiness (Cauvain, 2004).

\section{Structural features of bread}

Bread is produced through random physical processes which makes, its structure heterogeneous. Heterogeneity is expected to influence the mechanical behavior of bread
Table 4: Crumb features of bread made with JAP and leavened using co-culture

\begin{tabular}{lccc}
\hline Samples & $\begin{array}{c}\text { Cell density } \\
\left(1 / \mathbf{c m}^{2}\right)\end{array}$ & $\begin{array}{c}\text { Mean cell } \\
\text { size }\left(\mathbf{m m}^{2}\right)\end{array}$ & $\begin{array}{c}\text { Fraction of cell size } \\
\text { within total surface }(\%)\end{array}$ \\
\hline SC-0 (control) & $37.23 \pm 1.47 \mathrm{~b}$ & $1.22 \pm 0.02 \mathrm{a}$ & $45.39 \pm 1.58 \mathrm{a}$ \\
MC-0 & $45.96 \pm 4.49 \mathrm{a}$ & $0.99 \pm 0.09 \mathrm{~b}$ & $45.17 \pm 0.97 \mathrm{a}$ \\
MC-5 & $45.81 \pm 2.54 \mathrm{a}$ & $0.97 \pm 0.07 \mathrm{~b}$ & $44.08 \pm 1.55 \mathrm{ab}$ \\
MC-7.5 & $50.05 \pm 0.96 \mathrm{a}$ & $0.88 \pm 0.02 \mathrm{~b}$ & $44.22 \pm 0.72 \mathrm{ab}$ \\
MC-10 & $45.62 \pm 3.21 \mathrm{a}$ & $0.93 \pm 0.04 \mathrm{~b}$ & $42.19 \pm 1.96 \mathrm{~b}$ \\
\hline
\end{tabular}

Values are the mean of the three replicates \pm SD

crumbs. Using image analysis, the contribution of structural features to bread quality can be quantified (Zghal et al., 2002).

We performed image analysis of bread crumb to quantify a number of structural features such as cell density, mean cell size, and cell area fraction (Table 4). Fig. 4 shows the digital images of bread crumb before and after image processing was performed. The use of the co-culture significantly increased the cell density of non-enriched bread. This effect remained unaltered with increasing JAP. The mean cell size and cell area fraction of bread crumb were significantly affected by the use of the co-culture. Increased JAP had no effect on mean cell size, but decreased the cell area fraction, notably at a dose of $10 \%$ (Table 4).

JAP addition strongly influences the formation of cell walls constituting the crumb solids, in turn altering the dough elasticity. Furthermore, JAP addition impaired gas retention of the dough and resulted in a lower specific volume and cell area fraction (Rubel et al., 2015). The leavening agent (co-culture) manipulates crumb cell size and cell size distribution. Greater leavening ability produces greater cell size and lower cell density. Inversely, these factors produce smaller cell size and greater cell density. We previously demonstrated that $S$. cerevisiae had greater leavening ability than T. delbrueckii JK08 and P. anomala JK 04 (Wahyono et al., 2015). We also found that co-cultured $S$. cerevisiae and T. delbrueckii JK08 or P. anomala JK04 had lower leavening ability compared to $S$. cerevisiae alone, which resulted in greater cell density and smaller mean cell size (Wahyono et al., In press).

\section{Sensorial properties of bread}

Currently, instrumental measurement is the main method of evaluating the quality of food products. However, sensory attributes are also a key determinant in whether a product is acceptable for consumer needs. A considerable proportion of product failure can be attributed to a mismatch between sensory properties and consumer expectations (Kemp et al., 2009). Although consumer awareness regarding consumption of nutritious and healthy bread is increasing, producing such bread without compromising consumer acceptance is difficult. 


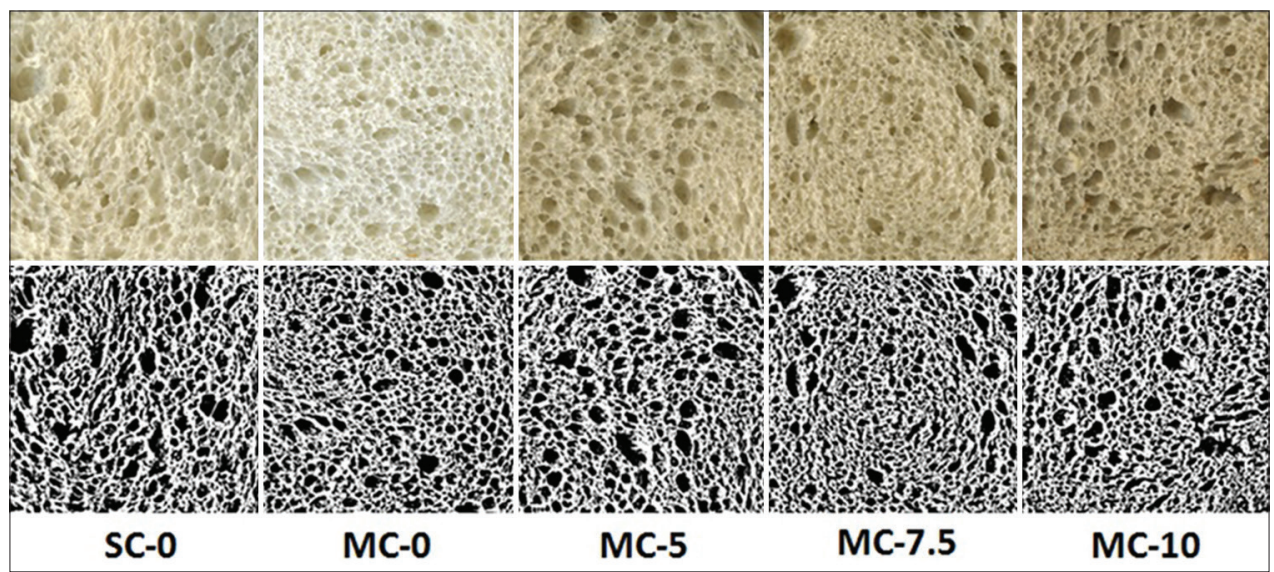

Fig 4. Digital images of JAP enriched bread before and after image processing

We performed sensory evaluation of bread enriched with JAP and leavened using co-culture of T. delbrueckii JK08 and P. anomala JK04. Generally, JAP-enriched breads leavened using co-culture showed satisfactorily scores in all sensory attributes (Fig. 5). The use of the co-culture noticeably improved the consumer acceptance of non-enriched bread (MC-0) in terms of texture, flavor, mouthfeel, and overall acceptability. For color attribute, the score was comparable to that of the control bread (SC-0). At low and moderate doses (5 and 7.5\%), co-culture improved the texture, flavor, mouthfeel, and overall acceptability, such that consumers scored these attributes as good as the control bread (SC-0). Worse scores were given to the 10\% (MC-10) JAP-enriched bread in terms of flavor, color, and texture which had scores of 3.44, 3.87, and 3.94, respectively. However, these values were statistically comparable to those of the control bread (SC-0), except for color. The color attributes of JAPenriched breads were noticeable to consumers. Previously, the instrumental color measurement could not detect the difference in the lightness and whiteness of crumb between the control and JAP-enriched bread. A noticeable color difference perceived by consumers between the two may have been because of a significant difference in crumb redness and yellowness. JAP-enriched bread generally had more intense reddish and yellowish crumb color.

Several studies have evaluated the sensory properties of inulin-enriched bread. Based on simple observation, the quality of bread made with 5\% JAP was unacceptable in all aspects. With 2.5\% JAP, consumers rated the bread comparable to the control bread in terms of texture, sponginess, and color (Rubel et al., 2015). Brasil et al. (2011) demonstrated that the use of $10 \%$ JAP impaired bread aroma, texture, and porosity. In addition, the bread enriched with $8 \%$ inulin was classified lower than the control bread due to wrinkling of the crust and the bread was darker and more intense in color (Korus et al., 2006). In contrast, Praznik et al. (2002) reported that the bread

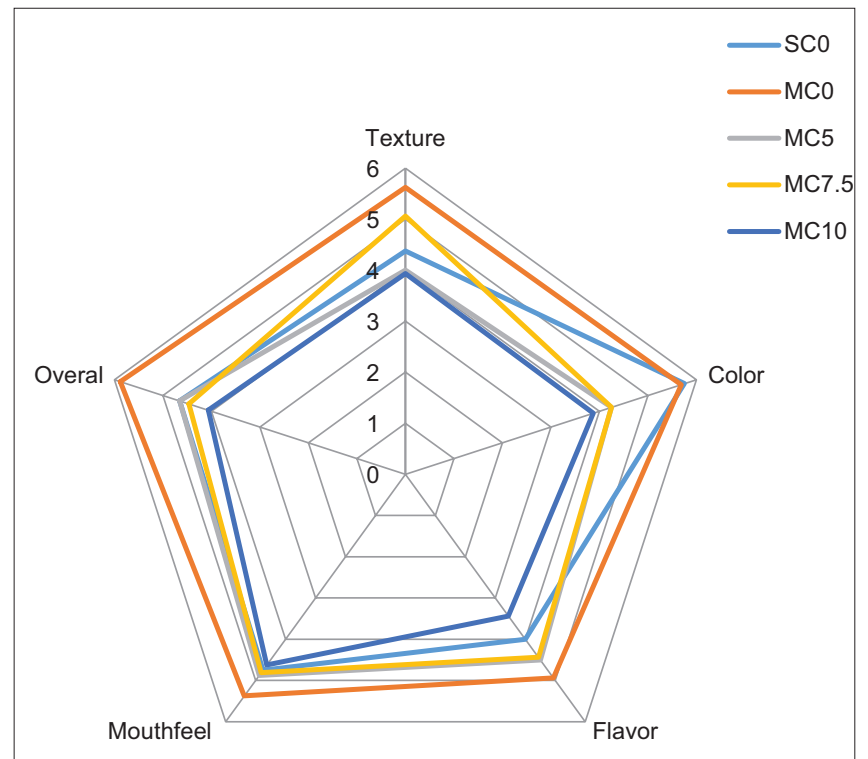

Fig 5. Radar plot of the sensory properties of JAP-enriched bread leavened with co-culture. The result reflects the means of scores from 15 semi-trained panelists

with 10 and $12 \%$ JAP were classified as good as standard bread. The high acceptance of substituted bread by the tester was because of the good color and taste due to the increase in Maillard reaction products.

\section{Correlation analysis of quality parameter of JAP- enriched bread}

There is a strong correlation between the physical properties and structural features or textural profiles of JAP-enriched bread leavened using a co-culture (Table 5). Crumb moisture was significantly negatively correlated with hardness and chewiness. It is well known that crumb hardening is strongly affected by the loss of moisture. Bread specific volume is also negatively related to hardness and chewiness. In this study, we demonstrated that a voluminous loaf generally had a greater mean cell size as well as cell area fraction, and 
Wahyono, et al.: Jerusalem artichoke powder enriched-bread

Table 5: Pearson correlation matrix of physical properties, structural features, and textural profiles of bread crumb made with JAP and leavened using co-culture

\begin{tabular}{lccccccc}
\hline Parameter & Moisture & Specific volume & Hardness & Chewiness & Springiness & Cohesiveness & Cell density \\
\hline Specific volume & 0.428 & & & & & & \\
Hardness & $-0.784^{* *}$ & $-0.787^{* *}$ & & & & & \\
Chewiness & $-0.772^{* *}$ & $-0.814^{* *}$ & $0.994^{* *}$ & & & & \\
Springiness & -0.485 & -0.464 & 0.437 & 0.496 & & & \\
Cohesiveness & 0.267 & -0.120 & -0.184 & -0.084 & 0.277 & & \\
Cell density & -0.412 & -0.393 & 0.411 & 0.477 & $0.757^{* *}$ & 0.438 & $-0.927^{* *}$ \\
Mean cell & $0.543^{*}$ & 0.496 & $-0.535^{*}$ & $-0.599^{*}$ & $-0.799^{* *}$ & -0.420 & -0.120 \\
Cell area fraction & $0.541^{*}$ & 0.406 & $-0.534^{*}$ & $-0.520^{*}$ & -0.215 & 0.188 & 0.434 \\
\hline
\end{tabular}

${ }^{*}$ Correlation is significant at the 0.01 level. ${ }^{\star}$ Correlation is significant at the 0.05 level

thus more pores (cells) than cellular solids. In contrast, a less voluminous loaf has a smaller cell size and cell area fraction (Table 4), resulting in greater hardness and chewiness. This is well defined by the negative correlation between hardness or chewiness with mean cell size and cell area fraction (Table 5). Additionally, springiness had a significant positive correlation to cell density. In contrast, springiness was negatively correlated with mean cell size. We previously found that springiness is related to the strength of the crumb cell wall network (Cauvain, 2004). Since the cell density showed a strong negative correlation to mean cell size, a greater mean cell size decreases cell density and vice versa. A greater mean cell size reduces the cellular solid connecting the crumb cell wall network, thus decreasing bread springiness.

\section{CONCLUSIONS}

Several studies have revealed that incorporating JAP or its derivatives had detrimental effects on bread quality and decreased consumer acceptance. Generally, at a lower dose (less than 5\%), there was no noticeable effect to the bread. In this study, we demonstrated that the use of a co-culture of T. delbrueckii JK08 and P. anomala JK04 in combination with commercial baker's yeast improved the quality of JAP-enriched bread at a higher dose $(5 \%$ or $7.5 \%)$. At these doses, the use of the co-culture improved specific volume, bread yield, crumb lightness, crumb whiteness, cell density, mean cell size, springiness, and cohesiveness. All sensory attributes were rated as good or better than the control bread except for color. In summary, the use of the co-culture clearly improved the quality of JAPenriched bread.

\section{ACKNOWLEDGEMENTS}

This study was financed by the Directorate General of Human Resources for Science, Technology and Higher Education, the Republic of Indonesia, and the Rural Development Administration, Republic of Korea (Research grant PJ009439022015).

\section{Authors contribution}

A. W. was involved in manuscript preparation, sample collection, conducting experiment and sample analysis, S. L. was involved in raw material analysis, S. Y. was involved in image analysis evaluation, W. K. was involved in designing study, H. P. was the project manager who designed study and did critical revisions.

\section{REFERENCES}

AACC 10-05. 2000. Guidelines for Measurement of Volume by Rapeseed Displacement. Approved Methods of the AACC, $10^{\text {th }}$ ed. American Association of Cereal Chemists, St. Paul.

AACC 54-21. 2000. Farinograph method for flour. Approved Methods of the AACC, $10^{\text {th }}$ ed. American Association of Cereal Chemists, St. Paul.

Annan, N.T., L. Poll, S.S. Dedeh, W.A. Plahar and M. Jakobsen. 2003. Influence of starter culture combinations of Lactobacillus fermentum, Saccharomyces cerevisiae and Candida krusei on aroma in Ghanaian maize dough fermentation. Eur. Food Res. Technol. 216: 377-384.

Atwell, W.A. 2001. Wheat and Flour Testing, American Association of Cereal Chemists, Ch. 4. Minnesota, Washington, D.C., Pp47-66.

Bartkiene, E., I. Jakobsone, G. Juodeikiene, D. Vidmantiene, I. Pugajeva and V. Bartkevics. 2013. Effect of fermented Helianthus tuberosus L. tubers on acrylamide formation and quality properties of wheat bread. LWT - Food Sci. Technol. 54: 414-420.

Brasil, J.A., K. Correia and S.M. Salgado. 2011. Effect of the addition of inulin on the nutritional, physical and sensory parameters of bread. Braz. J. Pharm. Sci. 47: 185-191.

Cauvain, S.P. 2004. Improving the texture of bread. In: Kilcast, D (Ed.), Texture in Food: Solid Foods, Wood Head Publishing, Cambridge, Pp435-448.

Cho, S.S. and P. Samuel. 2009. Fiber Ingredients: Food Application and Health Benefits, CRC Press, Boca Raton, pvii.

Czuchajowska, Z., Y. Pomeranz and H. Jeffers. 1989. Water activity and moisture content of dough and bread. Cereal Chem. 66: 128-132.

Elhariry, H.M., R.M. Mahmoud, A.A. Hassan and M.A. Aly. 2011. Development of co-culture sourdough systems for improving bread quality and delaying staling. Food Biotechnol. 25: 252-272.

Furlán, L.T.R., A.P. Padilla and M.E. Campderrós. 2015. Improvement of gluten-free bread properties by the incorporation of bovine plasma proteins and different saccharides into the matrix. Food Chem. 170: 257-264. 
Gunnarsson, I.B., S.E. Svensson, E. Johansson, D. Karakashev and I. Angelidaki. 2014. Potential of Jerusalem artichoke (Helianthus tuberosus L.) as a biorefinery crop. Ind. Crop Prod. 56: 231-240.

Hager, A.S., L.A.M. Ryan, C. Schwab, M.G. Gänzle, J.V. O'Doherty and E.K. Arendt. 2011. Influence of the soluble fibres inulin and oat $\beta$-glucan on quality of dough and bread. Eur. Food Res. Technol. 232: 405-413.

Hager, A.S., E. Zannini and E.K. Arendt. 2012. Formulating breads for specific dietary requirement. In: Cauvain, S.P. (Ed.), Bread Making: Improving Quality, CRC Press, Boca Raton, Cambridge, England. Pp. 711-735.

Hsu, C. 2003. Chemical composition, physical properties, and antioxidant activities of yam flours as affected by different drying methods. Food Chem. 83: 85-92.

Juszczak, L., T. Witczak, R. Ziobro, J. Korus, E. Cieslik and M. Witczak. 2012. Effect of inulin on rheological and thermal properties of gluten-free dough. Carbohyd. Polym. 90: 353-360.

Kays, S.J. and S.F. Nottingham. 2008. Biology and Chemistry of Jerusalem Artichoke. CRC Press, Boca Raton, USA.

Kemp, S.E., T. Hollowood and J. Hort. 2009. Sensory Evaluation: A Practical Handbook, Wiley-Blackwell, Chicester, United Kingdom.

Korus, J., K. Grzelak, K. Achremowicz and R. Sabat. 2006. Influence of prebiotic additions on the quality of gluten-free bread and on the content of inulin and fructooligosaccharides. Food Sci. Technol. Int. 12: 489-495.

Kosaric, N., G.P. Cosentino, A. Wieczorek and Z. Duvnjak. 1984. The Jerusalem artichoke as an agricultural crop. Biomass. 5: 1-36.

Lin, L.Y., H.M. Liu, Y.W. Yu, S.D. Lin and J.L. Mau. 2009. Quality and antioxidant property of buckwheat enhanced wheat bread. Food Chem. 112: 987-991.

Morris, C. and G.A. Morris. 2012. The effect of inulin and fructooligosaccharide supplementation on the textural, rheological and sensory properties of bread and their role in weight management: A review. Food Chem. 133: 237-248.

Movahed, S., G. Rooshenas and H.A. Chenarbon. 2012. Evaluation of the effect of yeast-salt method on dough yield, bread yield and organoleptic properties Iranian Lavash bread. Ann. Biol. Res. 3: 595-600.

O'Brien, C.M., A. Mueller, A.G.M. Scannell and E.K. Arendt. 2003. Evaluation of the effects of fat replacers on the quality of wheat bread. J. Food Eng. 56: 265-267.

Ogunbanwo, S.T., A.A. Adebayo, M.A. Ayodele, B.M. Okanlawon and M.O. Edema. 2008. Effects of lactic acid bacteria and Saccharomyces cerevisae co-cultures used as starters on the nutritional contents and shelf life of cassava-wheat bread. J. Appl. Biosci. 12: 612-622.

Peressini, D. and A. Sensidoni. 2009. Effect of soluble dietary fibre addition on rheological and bread making properties of wheat doughs. J. Cereal Sci. 49: 190-201.

Poinot, P., G. Arvisenet, J. Grua-Priol, C. Fillonneau, A. Le-Bail and C. Prost. 2010. Influence of inulin on bread: Kinetics and physicochemical indicators of the formation of volatile compounds during baking. Food Chem. 119: 1474-1484.

Praznik, W., E. Cieślik and A. Filipiak-Florkiewicz. 2002. Soluble dietary fibres in Jerusalem artichoke powders: Composition and application in bread. Nahrung. 46: 151-157.

Randez-Gil, F., P. Sanz and J.A. Prieto. 1999. Engineering baker's yeast: Room for improvement. Trends Biotechnol. 17: 237-244.

Roberfroid, M. 2002. Functional food concept and its application to prebiotics. Dig. Liver Dis. 34 Suppl 2: 105-110.

Rubel, I.A., E.E. Pérez, G.D. Manrique and D.B. Genovese. 2015. Fibre enrichment of wheat bread with Jerusalem artichoke inulin: Effect on dough rheology and bread quality. Food Struct. 3: 21-29.

Salovaara, H., Gate, F and M. Tenkanen. 2007. Dietary Fibre Components and Functions, Wageningen Academic, Netherland.

Skendi, A., C.G. Biliaderis, M. Papageorgiou and M.S. Izydorczyk. 2010. Effects of two barley $\beta$-glucan isolates on wheat flour dough and bread properties. Food Chem. 119: 1159-1167.

Stear, C.A. 1990. Handbook of Bread Making Technology, Elsevier, London, New York.

Ulziijargal, E., J.H. Yang, L.Y. Lin, C.P. Chen and J.L. Mau. 2013. Quality of bread supplemented with mushroom mycelia. Food Chem. 138: 70-76.

Wahyono, A., W.W. Kang and H.D. Park. 2015. Characterization and application of Torulaspora delbrueckii JK08 and Pichia anomala JK04 as baker's yeast. J. Food Nutr. Res. 54: 205-217.

Wahyono, A., S.B. Lee, W.W. Kang and H.D. Park. In Press. Improving bread quality using co-cultures of Saccharomyces cerevisiae, Torulaspora delbrueckii JK08, and Pichia anomala JK04. Ital. J. Food Sci.

Wang, J. 2002. Effect of the addition of different fibres on wheat dough performance and bread quality. Food Chem. 79: 221-226.

Zghal, M.C., M.G. Scanlon and H.D. Sapirstein. 2002. Cellular structure of bread crumb and its influence on mechanical properties. J. Cereal Sci. 36: 167-176. 